\title{
Collaborating to Improve Quality of Life in Primary Immunodeficiencies: World PI Week, 2013
}

\author{
Ricardo Sorensen • Amos Etzioni • \\ Ahmed Aziz Bousfiha • John B. Zeiger
}

Received: 16 April 2013 / Accepted: 24 June 2013 / Published online: 11 July 2013

(C) Springer Science+Business Media New York 2013

\section{Introduction}

World Primary Immunodeficiency Week (WPIW) 2013 ran from 22 to 29th April (www.worldpiweek.org), providing an opportunity to reflect on progress made and the challenges that remain for the primary immunodeficiency diseases (PID) community. In this article, representatives from leading PID societies evaluate the situation and plans for further progress in their respective areas of the world. It is hoped that this situation analysis will prompt further focus and enhanced collaboration on the gaps that remain in the pursuit of promoting early diagnosis and treatment of PIDs.

\section{Europe}

The European Society for Primary Immunodeficiencies (ESID; www.esid.org) is the oldest and largest professional organization focused on PIDs. Currently ESID has 800 members and almost 2,000 individuals attended its last biennial meeting (Florence, October 2012).

R. Sorensen $(\square)$

Department of Paediatrics, Children's Hospital,

200 Henry Clay Ave., New Orleans, LA, USA

e-mail: rsoren@1suhsc.edu

\section{A. Etzioni}

Meyer Children's Hospital, Haifa, Israel

\section{A. A. Bousfiha}

Clinical Immunology Unit, King Hassan II University,

Averroes Hospital, Casablanca, Morocco

J. B. Zeiger

Sydney Children's Hospital, Randwick, New South Wales, Australia
ESID developed the first clinical database for PIDs. Containing data from over 13,000 patients, this large registry has been used for many important projects and published studies [1-3]. Furthermore, in 2012, ESID members collaborated in the publication of an updated PID diagnostic protocol for nonimmunologists. This protocol incorporates newly defined PIDs and is designed to assist physicians caring for adults and children to promptly and cost-effectively screen and diagnose PIDs based on the clinical presentation and laboratory tests, with definitive tests subsequently performed in partnership with specialist immunologists [4].

Aside from organizing its biennial meeting, ESID is involved in many other educational activities, such as a summer school on PIDs. A major objective of the organization is to increase awareness of PIDs through a new website and meetings with patient organizations. ESID also supports the wonderful 'J project', conducted by Prof. László Maródi (Hungary), which aims to develop PID awareness and expertise in eastern and central European countries [5].

In the upcoming year, ESID will focus on two main points for Europe:

- Implementation of neonatal screening for severe combined immunodeficiency (SCID). After the success of the program in several states in the USA, ESID will push for SCID screening to be established soon in many European countries.

- Provision of adequate treatment of the various PIDs across Europe. Unfortunately, in many countries the supply of intravenous immunoglobulin (IVIG) is inadequate and haematopoietic stem cell transplantation (HSCT) centres have yet to be developed in some countries, mainly in eastern Europe.

Although much has been achieved, there is still a long way to go in providing the early and precise diagnosis of 
PID, together with the appropriate treatment, to the many thousands of patients all over Europe and the world.

\section{Latin America}

Organised efforts towards improving the diagnosis and management of patients with PIDs in Latin America started in 1993 when a small group of immunologists from Argentina, Brazil, Chile and Colombia teamed up with Ricardo Sorensen, M.D., in the USA to create a joint patient reporting form under the auspices of an informal group called the Latin American Group for Primary Immunodeficiencies (LAGID; www.lagid.lsuhsc.edu). The goals of this organization were to include other Latin American countries and to create registries of patients with PIDs in each participating country. The group grew from the initial four to a total of 14 countries with a wide population range.

Many of the objectives set forth by LAGID have been accomplished, including: the development of patient and parent support groups in most participating countries; the creation of a website for members; the organization of 12 yearly scientific meetings held from 1995 to 2007; educational programmes and the education of paediatricians and general practitioners; and extensive collaborative efforts between member countries and between Latin American immunologists and their colleagues in Europe and the US. These efforts led to the creation of the first joint PID registry published by eight countries in 1998 [6]. A second report was published in 2007 [7].

A second official electronic registry, following the model of the ESID registry, was established for all of Latin America in Sao Paulo, Brazil, with the assistance of ESID. Other actions have contributed to advancing the care of patients with PIDs in Latin America and have brought a new dynamic to diagnosis, treatment and research in the region $[8,9]$. These actions include the sponsored fellowship programme led by the Latin American Society for Immunodeficiencies (LASID; www.lasid.org), founded in 2009, which has assisted young immunologist in five countries to specialise in the diagnosis and care of PIDs, and the establishment of a network of Jeffrey Modell Diagnostic and Research Centers in Sao Paulo, Mexico City, Medellin, Chile and Buenos Aires. Created by the Jeffrey Modell Foundation (http:// www.info4pi.org/jmf/), these centres offer enhanced diagnostic and treatment capabilities for PID across the world, including now in Latin America. Health authorities in several countries, including Mexico, Colombia, Argentina, Brazil and Chile, have now recognised PIDs as a special group of diseases and facilitated access to treatment.

The present day challenges of PID in Latin America are two-fold: to continue to expand awareness and diagnosis and treatment possibilities to more areas in Latin America where there has been little or no progress, and to satisfy the desire of young Latin American immunologists to bring state-of-theart advances in diagnosis and treatment to their patients. The encouraging experience gained from years of successful collaborative efforts allows LASID to look with great optimism to its future.

\section{Africa and Middle East}

Africa has been late in developing PID diagnosis and care, but substantial progress has been made over the past 2 years. The African Society for Immunodeficiencies (ASID; www. asid.ma), created in Casablanca in 2008, has undertaken regional actions to educate paediatricians and other healthcare professionals and to promote cross-country initiatives to allow the exchange of expert experience and education. ASID has raised awareness of PIDs among physicians and the general public in African, especially in North Africa and South Africa, and its achievements are already palpable. Africa has a spokesperson for PID scientific and social societies, and schools to provide education about PIDs have involved a dozen countries in each region of Africa. ASID has recently hosted its 3rd International Congress (Sun City, South Africa, June 5-9, 2013) where the 5th PID School took place. In addition, ASID has begun to collect data with the launch of an international registry. At the regional level, ASID has allowed the creation or consolidation of PID networks and the publication of multicentre studies on these conditions. In particular, two workshops bringing together experts from North Africa and the Middle East were held in Athens in 2012 and in Dubai in 2013. These led to the sharing of solutions between experts and to the building of cooperative bridges in training and research. The Moroccan Society for Primary Immunodeficiencies, the Hajar Association and the Faculty of Sciences of Ben M'sik University (Casablanca) have also collaborated to provide PID training, including the 1st PID Spring School held in April. With regard to research, in 2011, Ridha Barbouche and colleagues published a profile of PIDs in North Africa (Morocco, Tunisia and Egypt) [10], while Saleh Al Muhsen and colleagues published on the epidemiology of PID in the Middle East [11]. Both regions are characterised by high rates of inbreeding (20-60\%) and they have a comparable distribution of PID causes, with a high incidence of SCID and founder mutations. Teams in the Middle East, including Saudi Arabia, have published excellent results on HSCT and have contributed to internationals research on PID specific cases [12].

However, substantial challenges remain in Africa, whose population has this year exceeded one billion citizens. In North Africa, difficulties exist in IVIG access and HSCT has been introduced only timidly. The Arabic revolution (Printemps Arabe) triggered a mobilization of citizens with regard to the 
quality of care and the reimbursement of costly treatments, such as IVIG. For example, in Morocco, the new government launched the RAMED program (medical assistance program to disadvantaged people) and reinforced the AMO (Obligatory Medical Assurance; AMO programme), which requires hospitals to have immunoglobulin and to reimburse care for PID patients more commonly. These advances are welcome and they provide hope for patients with PIDs in this region. However, with the exception of South Africa-where the situation is comparable to that in North Africa-progress is lagging in other African countries. Indeed, there seems to be little or no activity in the PID field in over 40 of the 54 countries of this continent. The burden of HIV infection, and other epidemics, in Sub-Saharan Africa is most likely overlapping and obscuring the PID burden of diseases.

Urgent international actions are therefore necessary, particularly in Sub-Saharan Africa, since PID is still largely underdiagnosed. Indeed, PIDs are more common than is generally thought. It is estimated that up to 900,000 people in Africa may have a PID, whereas only around 1,000 cases are currently registered [13]. These international actions should aim not only to increase awareness of PIDs among physicians, nurses and other healthcare professionals, but in particular to bring the attention of the authorities, including the WHO, and the general public to this large group of rare diseases and their significant effect on infant mortality. Local PID working groups in each country are best placed to address local authorities and the general public and ASID aims to establish such groups where these do not exist at present. The assistance of international experts is required in this process, to help raise awareness of PIDs among the medical community and then to train members of local working groups.

In conclusion, international collaboration is the only way to improve PID awareness, diagnosis and care in Africa. The two regions of North Africa and the Middle East have rightly decided to work together to improve PID diagnosis and care. Corresponding joint efforts are also needed in the rest of Africa to enable the work of patient groups, doctors and nurses to bring a positive change for PID patients.

\section{Australia}

There is an active immunodeficiency community in Australia, with many of the major interactions revolving around the PID Committee of the Australasian Society of Clinical Immunology and Allergy (ASCIA; www.allergy.org.au) and the now well-established triennial Asia Pacific Primary Immune Deficiency (APPID) Summer School (www.appid. org). While geographical constraints make large PID centres difficult to envisage, there are a number of major centres seeing patients with complex immunodeficiency disorders and the first Australian Jeffrey Modell Foundation Center will soon open at Sydney Children's Hospital, Randwick. The outcomes amongst the five centres currently performing HSCT for PIDs in Australia compare favourably to those from other parts of the world [14].

From a research perspective there has been an effort to form collaborative research partnerships across the country, initially through the Australian PID registry [15] and more recently through the antibody deficiency orientated Australia and New Zealand Antibody Deficiency Allele (ANZADA) study, which has now being joined by the more paediatricorientated CIRCA group.

As in other (but not all) countries with similar economies, IVIG use is increasing by more than $10 \%$ per annum. In Australia most IVIG use is funded by the federal government [16] and distributed through the Red Cross Blood Service. A study in New South Wales is interrogating the Red Cross database to improve our understanding of the factors driving these changes. Subcutaneous immunoglobulin (SCIG) is becoming increasing popular among PID patients. SCIG appears to require lower overall immunoglobulin doses and some centres report that more patients are now using SCIG rather than IVIG. Although three products have been approved by regulatory authorities for SCIG use, and SCIG has been endorsed in the Australian criteria for the clinical use of immunglobulin (http://www.nba.gov.au/ivig/index. html), national funding of SCIG use is still awaited.

\section{Conclusion}

The last 10 years has seen an unprecedented improvement in PID care in many areas of the world, including some areas where provision was seriously lacking. These improvements have resulted from collaborative efforts involving immunologists, non-specialist physicians, nurses' associations, health providers, policymakers, and patient organisations. Such collaborations offer a model for further efforts to promote early diagnosis and therapy in order to extend and improve the lives of people with PIDs, especially in areas of greatest need.

\section{References}

1. Eades-Perner AM, Gathmann B, Knerr V, Guzman D, Veit D, Kindle G, et al. The European internet-based patient and researchdatabase for primary immunodeficiencies: results 2004-06. Clin Exp Immunol. 2007;147:306-12.

2. Gathmann B, Grimbacher B, Beauté J, Dudoit Y, Mahlaoui N, Fischer A, et al. The European internet-based patient and researchdatabase for primary immunodeficiencies: results 2006-2008. Clin Exp Immunol. 2009;157 Suppl 1:3-11.

3. Gathmann B, Binder N, Ehl S, Kindle G, ESID Registry Working Party. The European internet-based patient and research database for 
primary immunodeficiencies: update 2011. Clin Exp Immunol. 2012;167:479-91.

4. de Vries E. European Society for Immunodeficiencies (ESID) members. Patient-centred screening for primary immunodeficiency, a multi-stage diagnostic protocol designed for non-immunologists: 2011 update. Clin Exp Immunol. 2012;167:108-19.

5. Maródi L, J Project Study Group. The creation and progress of the J Project in Eastern and Central Europe. Ann NY Acad Sci. 2011;1238:65-73.

6. Zelazko M, Carneiro-Sampaio M, CornejodeLuigi M, GarciadeOlarte D, PorrasMadrigal O, BerrónPerez R, et al. Primary immunodeficiency diseases in the Latin America: first report from eight countries participating in LAGID. J Clin Immunol. 1998;18:161-6.

7. Leiva LE, Zelazco M, Oleastro M, Carneiro-Sampaio M, CondinoNeto A, Costa-Carvalho BT, et al. Primary immunodeficiency diseases in Latin America: the second report of the LAGID registry. J Clin Immunol. 2007;27:101-8.

8. Errante PR, Franco JL, Espinosa-Rosales FJ, Sorensen R, CondinoNeto A. Advances in primary immunodeficiency diseases in Latin America: epidemiology, research, and perspectives. Ann N Y Acad Sci. 2012;1250:62-72.

9. Condino-Neto A, Franco JL, Espinosa-Rosales FJ, Leiva LE, King A, Porras $\mathrm{O}$, et al. Advancing the management of primary immunodeficiency diseases in Latin America: Latin American Society for Immunodeficiencies (LASID) Initiatives. Allergol Immunopathol (Madr). 2012;40:187-93.
10. Barbouche MR, Galal N, Ben Mustapha I, Jeddane L, Mellouli F, Ailal F, et al. Primary immunodeficiencies in highly consanguineous North African populations. Ann NY Acad Sci. 2011;1238:42-52.

11. Al-Muhsen S, Alsum Z. Primary immunodeficiency diseases in the Middle East. Ann N Y Acad Sci. 2012;1250:56-61.

12. Hassan A, Booth C, Brightwell A, Allwood Z, Veys P, Rao K, et al. Outcome of hematopoietic stem cell transplantation for adenosine deaminase-deficient severe combined immunodeficiency. Blood. 2012;120:3615-24.

13. Bousfiha AA, Jeddane L, Ailal F, Benhsaien I, Mahlaoui N, Casanova JL, et al. Primary immunodeficiency diseases worldwide: more common than generally thought. J Clin Immunol. 2013;33:1-7.

14. Mitchell R, Nivison-Smith I, Anazodo A, Tiedemann K, Shaw P, Teague L, et al. Outcomes of hematopoietic stem cell transplantation in primary immunodeficiency: a report from the Australian and New Zealand Children's Haematology Oncology Group and the Australasian Bone Marrow Transplant Recipient Registry. Biol Blood Marrow Transplant. 2013;19:338-43.

15. Kirkpatrick P, Riminton S. Primary immunodeficiency diseases in Australia and New Zealand. J Clin Immunol. 2007;27:517-24.

16. National Blood Authority National Report on the Issue and Use of Intravenous Immunoglobulin (IVIg) for 2010-2011. Canberra; National Blood Authority. www.nba.gov.au/ivig/ivig-report-10-11_ amended_dec2012.pdf. Accessed 5 March 2013 